generation of targeted antimicrobial therapies for diseases that are currently difficult to treat. Indeed, there might be many diseases that we currently don't realize have a microbial component, and which could be tackled by phages.

Martha R. J. Clokie is in the Department of Genetics and Genome Biology, University of Leicester, Leicester LE1 7RH, UK. e-mail: mrjc1@leicester.ac.uk

1. Marshall, B. J., Armstrong, J. A., McGechie, D. B. \& Glancy, R. J. Med. J. Aust. 142, 436-439 (1985).

. Duan, Y. et al. Nature 575, 505-511 (2019).

3. Llorente, C. et al. Nature Commun. 8, 837 (2017)

4. Van Tyne, D. \& Gilmore, M. S. Annu. Rev. Microbiol. 68, 337-356 (2014).
5. Lebreton, F. et al. Cell 169, 849-861 (2017).

6. Ubeda, C. et al. J. Clin. Invest. 120, 4332-4341 (2010).

7. Bolocan, A. S. et al. Viruses 11, 366 (2019).

8. Arias, C. A. \& Murray, B. E. Nature Rev. Microbiol. 10 266-278 (2012).

9. Nguyen, S. et al. mBio 8, e01874-17 (2017).

10. Abedon, S. T., Kuhl, S. J., Blasdel, B. G. \& Kutter, E. M. Bacteriophage 1, 66-85 (2011).

11. Nale, J. Y., Chutia, M., Carr, P., Hickenbotham, P. T. \& Clokie, M. R. J. Front. Microbiol. 7, 1383 (2016).

12. Nale, J. Y., Redgwell, T. A., Millard, A. \& Clokie, M. R. J. Antibiotics 7, 13 (2018).

13. Whiteson, K. L. mSystems 3, 00166-17 (2018)

14. Melo, L. D. R., Ferreira, R., Costa, A. R., Oliveira, H. \& Azeredo, J. Sci. Rep. 9, 6643 (2019).

15. Barros, J. et al. Int. J. Antimicrob. Agents 54, 329-337 (2019).

16. Al-Zubidi, M. et al. Infect Immun. 87, e00512-19 (2019).

17. del Rio, B. et al. Front. Microbiol. 10, 566 (2019).

This article was published online on 13 November 2019

\title{
Engineering
}

\section{Virtual and augmented reality enhanced by touch}

\section{Xiao-ming Tao}

Conventional technologies for virtual and augmented reality simulate interactive experiences through visual and auditory stimuli. A technology that adds sensations of touch could find uses in areas from gaming to prosthetic feedback. See p.473

Human sensation includes the commonly known senses and less-recognized ones such as thirst, hunger and balance. Stimuli detected by sensory receptors are encoded into electrical signals that move along neural pathways to specific parts of the brain to be decoded into useful information. The whole process is complex. For instance, the sense of touch is a collection of several sensations, encompassing pressure, pain and temperature, and touch receptors are stimulated by a combination of mechanical, chemical and thermal energy. Until now, it has been a great challenge to incorporate sensations of touch into virtual and augmented reality. But on page 473 , Yu et al. report a skinintegrated technology that applies pressure, vibration or motion to the user, enabling communication between the user and a machine for virtual and augmented reality (X. Yu et al. Nature 575, 473-479; 2019).

The authors' technology consists of a soft, lightweight sheet of electronics that adheres to skin, and conforms to the body's shape, in a convenient, non-invasive and reversible manner (Fig. 1). The sheet contains arrays of vibratory actuators - mechanical components that convert electrical energy into vibrations. Each actuator comprises two connected parts: a coil of copper wire sealed in an acrylic base, and a permanent magnet mounted on a polymer beam. When an electric current passes through the coil, the magnet vibrates at the same frequency as that of the current.

Each actuator has a mass of only 1.4 grams and is millimetre-sized (12-18 $\mathrm{mm}$ in diameter
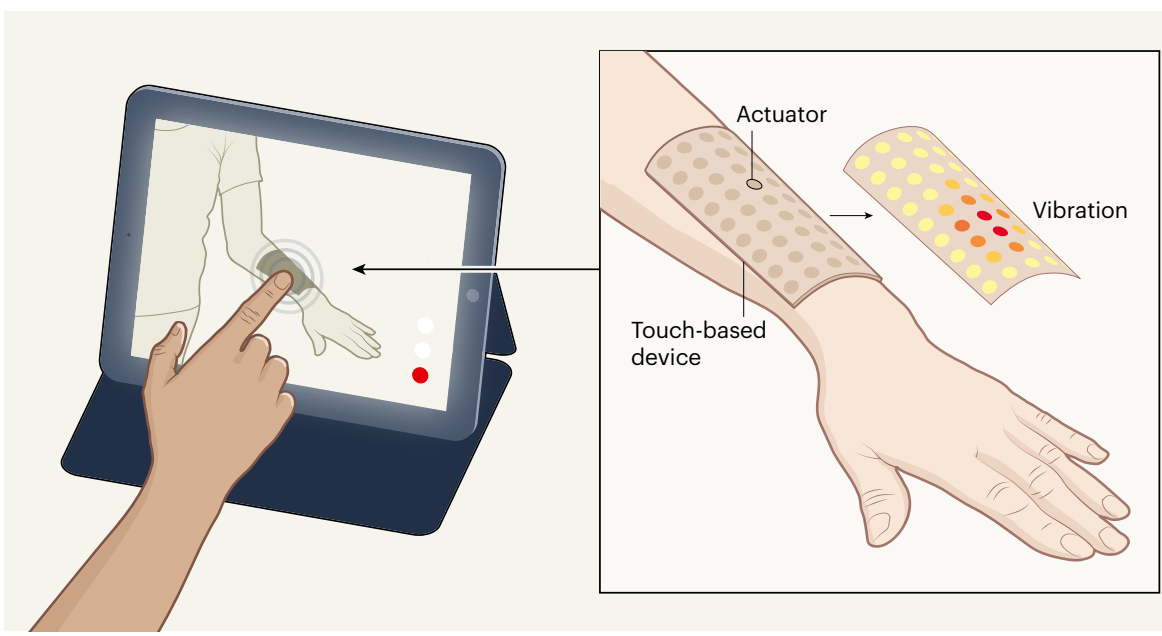

Figure 1 | Sense of virtual touch. Yu et al. present a device for incorporating touch-based sensations in virtual and augmented reality. The device consists of a lightweight sheet of electronics that softly laminates onto the skin. In this simple example, a touch screen displays a video feed of a person wearing the device, and a second person touches the image of the device on the screen. Mechanical components called vibrational actuators apply vibrations to the skin of the person wearing the device, providing a sense of virtual touch. The colours of the actuators represent their degree of activation from low (yellow) to high (red). 


\section{News \& views}

example, a person playing a combat-based video game wears several devices across their body; the devices are activated when a strike occurs on the corresponding body part of the game character.

The technology does have some drawbacks. For instance, each actuator is driven by a set current of about 5 milliamps, which is relatively high compared with that found in other consumer digital electronics. In addition, the energy lost from the other components as heat might affect actuator performance and cause warming of the skin if dissipation of the heat is not well managed. Moreover, although an optimized actuator requires only 1.75 milliwatts of power, the overall power consumption of the technology is still a key limiting factor in operating the platform sustainably and wirelessly for practical use. Miniaturization of the actuators could be a feasible way to address these issues, as the authors point out.

In 2002, many people were inspired by

\section{"The device is less cumbersome than wearable technologies that require connecting wires or internal batteries."}

a smart wearable invention known as the Hug Shirt, which allows hugs to be sent over a distance with the same ease as sending a text message or chatting (see go.nature. com/32kg1oz). This technology is equipped with embedded sensors that detect and encode the strength, duration and location of the touch, together with the skin warmth and heart rate of the sender. Through wireless communication and a control circuit, these signals are decoded to control actuators that reproduce the sensation of the hug for the receiver. Both the Hug Shirt and Yu and colleagues' device suggest that the application of touch sensations in virtual and augmented reality is just beginning, and that more exciting progress can be expected in the future.

Xiao-ming Tao is at the Research Centre for Smart Wearable Technology, Institute of Textiles and Clothing, Hong Kong Polytechnic University, Kowloon, Hong Kong.

e-mail: xiao-ming.tao@polyu.edu.hk

\section{nature}

podcast

\section{The week's best science, from the world's leading science journal.}

\section{NATURE.COM/NATURE/PODCAST}

